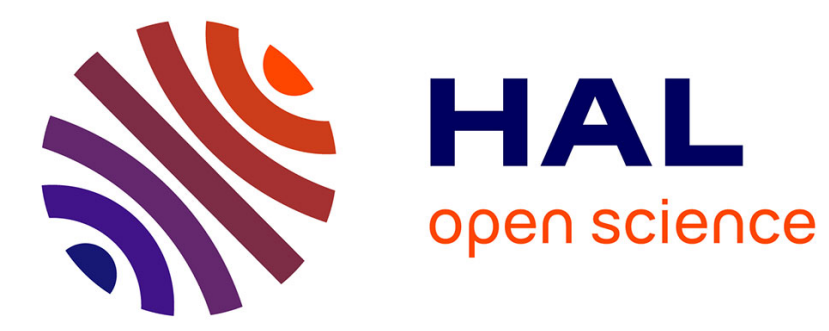

\title{
Les figures selon les scholies à Homère
}

Françoise Letoublon

\section{To cite this version:}

Françoise Letoublon. Les figures selon les scholies à Homère. Sandrine Dubel; Anne-Marie FavreauLinder; Estelle Oudot. Homère rhétorique. Études de réception antique, p. 121-139, 2018. hal02098905

\section{HAL Id: hal-02098905 \\ https://hal.univ-grenoble-alpes.fr/hal-02098905}

Submitted on 13 Apr 2019

HAL is a multi-disciplinary open access archive for the deposit and dissemination of scientific research documents, whether they are published or not. The documents may come from teaching and research institutions in France or abroad, or from public or private research centers.
L'archive ouverte pluridisciplinaire HAL, est destinée au dépôt et à la diffusion de documents scientifiques de niveau recherche, publiés ou non, émanant des établissements d'enseignement et de recherche français ou étrangers, des laboratoires publics ou privés. 


\title{
LES FIGURES SELON LES SCHOLIES À HOMÈRE
}

\author{
Françoise LETOUBLON ${ }^{1}$
}

Le présent article se situe dans le cadre général d'une recherche collective sur les scholies à Homère à laquelle le centre homérique souhaitait donner une impulsion. L'initiative "Homère rhétorique » était l'occasion idéale pour tenter de faire le point dans ce domaine, à partir des figures de la rhétorique ${ }^{2}$ : quelles sont les figures connues des scholiastes et repérées explicitement par eux dans les scholies homériques ? Parmi ces figures, la concentration sur les métaphores et comparaisons correspond à certains problèmes qui m'ont paru cruciaux dans l'analyse du texte homérique. Les ouvrages de référence sont dans ce domaine l'édition des scholies de l'Iliade par Hartmut Erbse, tandis que les scholies de l'Odyssée n'ont pas reçu d'édition moderne, celle de Dindorf remontant à 1855. On trouvera dans la bibliographie les références aux études critiques de Van der Valk, Kennedy, Pfeiffer, Nagy, Rengakos, Schmidt, et plus récemment Dickey, qui propose un panorama très complet des études homériques, du moins dans le monde anglophone. Avec le délai intervenu entre la remise de cet article et la réception des épreuves, il faut maintenant ajouter les volumes publiés sous l'égide de Franco Montanari chez De Gruyter et chez Brill ${ }^{3}$.

\section{QUELLES SONT LES FIGURES CONNUES DES SCHOLIASTES ?}

Le nom des figures est bien connu des spécialistes de rhétorique, avec un petit problème dès l'abord puisqu'au terme latin unique figura semblent correspondre en grec deux mots, $\tau \rho o ́ \pi о \varsigma$ et $\sigma \chi \tilde{\eta} \mu \alpha^{4}$. Il n'est pas question de nous engager dans le grand débat sur la valeur des deux termes et une éventuelle différence.

La lecture extensive des scholies repérées à partir de ces deux entrées permet de remarquer la fréquence très importante, pour les deux termes, de différents modèles de

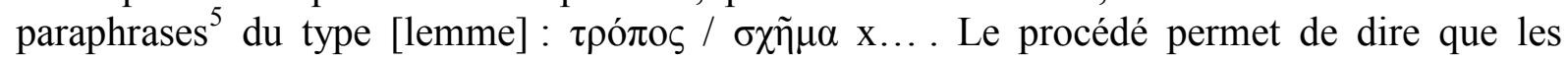
scholiastes répertorient les figures sous le terme générique de $\tau \rho o ́ \pi o \varsigma$ ou de $\sigma \chi \tilde{\eta} \mu \alpha$ en précisant ensuite le nom spécifique de chacune. En voici quelques exemples qui m'ont paru significatifs, avec l'emploi du verbe « appeler » dans deux cas, par simple juxtaposition dans un troisième :

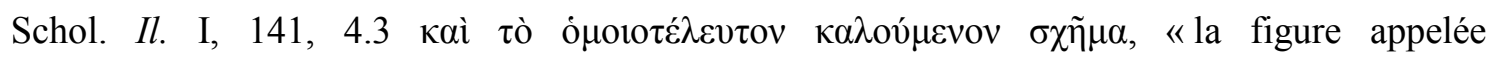
homeotéleute»;

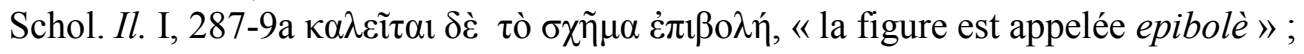

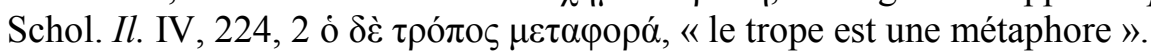

\footnotetext{
1 Professeur de littérature grecque à l'université Stendhal-Grenoble 3.

${ }^{2}$ Dans le prolongement du colloque organisé par Maria Silvana Celentano, Pierre Chiron et Marie-Pierre Noël, publié aux Éditions Rue d'Ulm sous le titre $\Sigma \chi \tilde{\eta} \mu \alpha$ / Figura.

${ }^{3}$ From Scholars to Scholia. Chapters in the History of Ancient Greek Scholarship, ed. Franco Montanari, Lara Pagani, Berlin/New York, 2011, et Brill's Companion to Ancient Greek Scholarship, ed., Franco Monanari, Stefanos Matthaios, Antonios Rengakos, Leiden/Boston, 2015.

4 M. Casevitz, «Étude lexicologique. Du schèma au schématisme », se limite au second terme, sans évoquer la relation au premier.

${ }^{5}$ Le procédé de paraphrase est analysé du point de vue de la linguistique générale par C. Fuchs, La Paraphrase et Paraphrase et énonciation.
} 


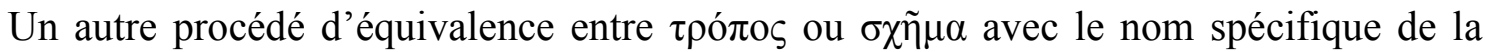
figure au génitif confirme cette hypothèse, par ex. Schol. Il. I, 366a $\tau \rho o ́ \pi o v$

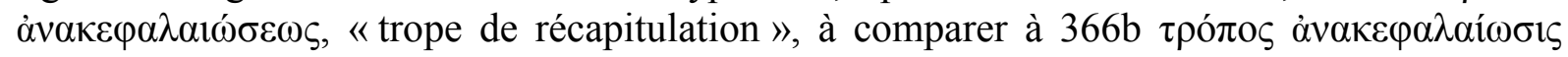
(idem).

De ces paraphrases, il semble qu'on peut déduire que les figures sont rangées par genres et par espèces, suivant le principe des classifications aristotéliciennes ${ }^{6}$.

Nous nous contenterons de prendre les occurrences de ces termes sans chercher à les distinguer puisque, pour les scholiastes, on a l'impression que les deux catégories sont synonymes et correspondent à la même liste de procédés rhétoriques ${ }^{7}$. Il est vrai que Quintilien $^{8}$, de son côté, semble distinguer entre $\sigma \chi \tilde{\eta} \mu \alpha$, traduit en latin par figura, et $\tau \rho$ ó $\mathrm{co} \varsigma$, traduit, si l'on ose dire, par tropus. Mais cela ne nous éclaire guère : il faudrait étudier la nuance qu'il établit entre les deux termes... Par ailleurs, et cela se trouve peut-être aussi dans les scholies homériques même si je n'en ai pas rencontré d'exemples dans le cadre limité de la présente recherche, $\sigma \chi \tilde{\eta} \mu \alpha$ signifie aussi « forme $^{9}$ », $\tau \rho$ ó $\pi$ o « «tour, expression » : pour faire court, les deux mots ont un spectre d'emplois différent, mais se recouvrent partiellement dans le sens rhétorique. Le fait que l'on trouve la plupart des figures sous les deux termes génériques peut s'interpréter soit en fonction d'une distinction théorique en grec, probablement chez les stoïciens, antérieurement à l'emprunt de Quintilien, soit selon une confusion faite par les scholiastes, comme j'incline à le croire sans avoir pu approfondir la question.

Je m'attacherai plus particulièrement dans la suite de l'étude aux procédés de transposition, comparaison et métaphore, en justifiant ce choix par le fait qu'ils occupent une position centrale dans la poétique d'Homère et la poétique grecque dans son ensemble : $\mathrm{j}$ 'en prends à témoin le Traité $d u$ sublime dans l'Antiquité ${ }^{10}$, puis pour les modernes Fränkel et Stanford ${ }^{11}$.

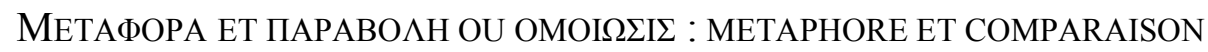

On trouve 103 occurrences de $\pi \alpha \rho \alpha \beta o \lambda \eta ́, 251$ de $\mu \varepsilon \tau \alpha \varphi \rho \rho \alpha ́$ dans le corpus des scholies

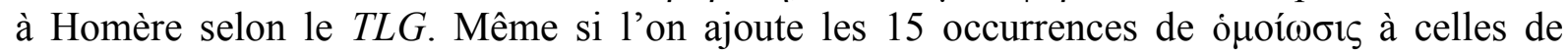
$\pi \alpha \rho \alpha \beta 0 \lambda \eta ́$, on voit que la très grande majorité des commentaires comportent le nom de la métaphore. Le rapport numérique semble curieusement inverse à l'emploi des deux figures dans le texte homérique ${ }^{12}$ : on connaît l'importance des comparaisons, à la fois du point de

\footnotetext{
${ }^{6}$ Sur le rôle de la classification dans l'approche scientifique des phénomènes selon Aristote, voir M. Crubellier et P. Pellegrin, Aristote, le philosophe et les savoirs, p. 97-109; sur la rhétorique, p. 142-149.

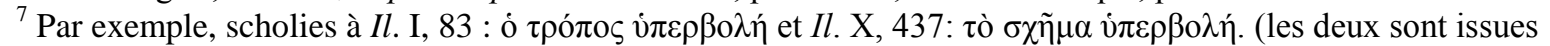
des scholia recentiora Theodori Meliteniotis e cod. Genevensi gr. 44)

${ }^{8} \mathrm{Au}$ livre VIII sur les tropes, IX sur les figures, cf. IX, 1 : l'ironie, par exemple, est « tantôt un trope et tantôt une figure $\gg$.

${ }^{9}$ C. Sandoz, Les Noms grecs de la forme. Voir aussi M. Casevitz, «Étude lexicologique. Du schèma au schématisme ».

${ }^{10}$ Voir la contribution de S. Conte dans le présent volume.

${ }^{11}$ Les études plus récentes sur les images homériques prennent moins souvent en compte les commentaires anciens. Par exemple, W.C. Scott, The Oral Nature of the Homeric Simile, ne les mentionne nullement. M. Edwards en note parfois, par ex. Aristarque à propos d'Il. XVI, 364, The Iliad : A Commentary, p. 35, n. 40.

${ }^{12} \mathrm{M}$. W. Edwards a regardé chez les divers spécialistes du domaine comment on a dénombré les comparaisons homériques dans l'histoire de la critique, et a noté les divergences d'un auteur à l'autre : D. Lee, The Similes of the Iliad and the Odyssey compared, compte 197 comparaisons développées « complètes », c'est-à-dire avec une forme verbale, dans l'Iliade, et 153 " courtes », alors qu'il en compte 45 et 87 respectivement dans l'Odyssée. W.C. Scott, The Oral Nature of the Homeric Simile, compte au total 341 comparaisons (deux types cumulés) dans l'Iliade, 134 pour l'Odyssée. A. Bonnafé, «Quelques remarques à propos des comparaisons homériques de
} 
vue numérique et par l'ampleur extraordinaire que donne l'aède à certaines d'entre elles, spécialement dans des moments où le détour poétique semble pour un regard naïf contraire aux impératifs de l'urgence narrative. La poétique de l'épopée semble reposer essentiellement sur les comparaisons ; en revanche les métaphores chez Homère semblent « figées » (voir les

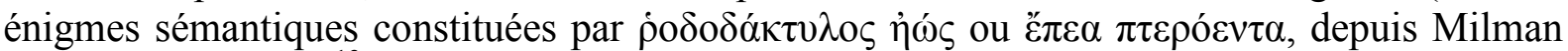
Parry, Durante, etc. $\left.{ }^{13}\right)$. Les scholiastes utilisent aussi souvent la forme verbale $\varepsilon i \kappa a ́ \zeta \varepsilon 1, ~ «(l e$ poète) compare ", qui permet peut-être de ne pas choisir entre comparaison et métaphore, ou contribue à rétablir dans les scholies l'équilibre numérique entre les deux termes que l'on peut attendre à partir du texte commenté.

Je choisis ci-après quelques exemples, sans idée préconçue, et en m'appuyant surtout sur les cas où j'ai eu l'impression de comprendre à peu près le style, difficile à mes yeux, des scholiastes, pour lequel il me semble qu'il faut un long temps de lecture passive, d'accoutumance, avant de commencer à entrer dans la perspective qui est la leur.

Une scholie à $I l$. II, 87 attribuée à Aristonicus ${ }^{14}$ commente une comparaison de combattants à un essaim d'abeilles, d'autant plus importante que, comme le scholiaste le remarque lui-même, c'est la première comparaison de l'Iliade ${ }^{15}:$ il semble que sa curiosité ait été déclenchée par l'application, apparemment " métaphorique », du terme $\varepsilon \theta v \varepsilon \alpha$ aux abeilles, à la place du terme attendu $\sigma \mu \eta ́ v \varepsilon \alpha$, « essaim », que justement le texte n'emploie pas. La comparaison commence donc par une sorte de préparation par l'application au monde animal d'un terme attendu pour le monde humain, à l'inverse de ce que l'on attend d'ordinaire spontanément $^{16}$ :

\section{II."87a".1}

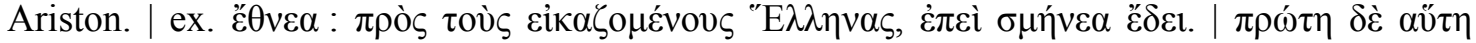

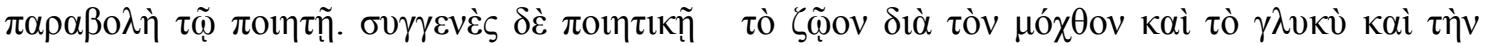

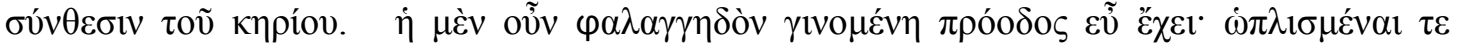

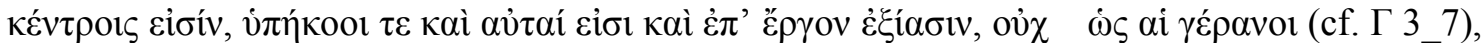

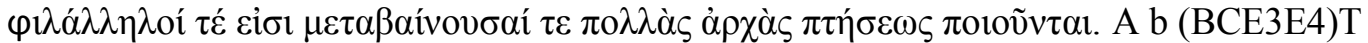

$\varepsilon \ddot{\theta} \theta v \varepsilon \alpha$ : à propos des Grecs qui sont l'objet de la comparaison, car il aurait fallu parler d'essaims. Cette comparaison est pour le poète la première. L'animal a une parenté avec eux du point de vue poétique par la peine qu'il se donne, par la douceur et par la constitution du rayon de miel. La sortie qui se produit par phalanges est bonne ; les abeilles sont armées de leur dard, et elles sont soumises et sortent pour travailler, nullement comme les grues (cf. Il. III, 3-7), mais en entente mutuelle et en se succédant pour le commandement du vol.

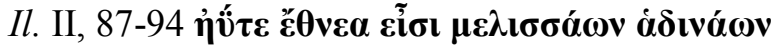

l'Iliade », a calculé le pourcentage des comparaisons par rapport au texte : 1128 vers sur 15693 vers pour l'Iliade, soit 7,2 \% du total (M. Edwards, The Iliad: A Commentary, p. 24 sq.).

${ }^{13}$ Voir les références dans F. Létoublon, « Comparaisons et métaphores ».

${ }^{14}$ Sur ce scholiaste, voir F. Razzetti, « Aristonicus », in Lessico dei Grammatici Greci Antichi, s. v. , L. Pagani, «Pioneers of Grammar. Hellenistic Scholarship and the Study of Language », in Montanari - Pagani, 2011, p. 17-64, ; S. Matthaios, « Greek Scholarship in the Imperial Era and Late Antiquity », in Montanari-MatthaiosRengakos, 2015, p. 220

${ }^{15}$ Dans l'optique d'une lecture continue du texte tel qu'il se présente dans la Vulgate bien entendu. Pourtant, comme le montre son appendice, W. C. Scott, The Oral Nature of the Homeric Simile, p. 191, voit quatre comparaisons dans le chant I : v. 47 « il allait semblable à la nuit», 104 «ses yeux ressemblaient à un feu éclatant », 265 « semblable aux immortels » (vers suspecté d'interpolation parce qu'il s'agit de Thésée) et 359 « comme une nuée », à propos de Thétis. Ces comparaisons brèves ne méritent apparemment pas pour les scholiastes le statut de comparaison homérique au sens plein du terme.

${ }^{16}$ Sur les comparaisons des humains à des animaux, voir W. C. Scott, The Oral Nature of the Homeric Simile, A. Schnapp-Gourbeillon, Lions, héros, masques, et S. H. Lonsdale, Creatures of Speech. 


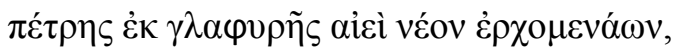

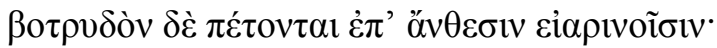

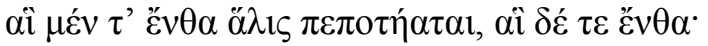

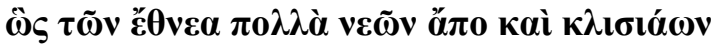

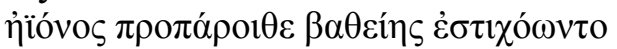

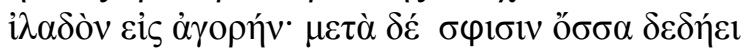

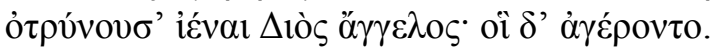

Comme on voit les abeilles, par troupes compactes, sortir d'un antre creux, à flots toujours nouveaux, pour former une grappe, qui bientôt voltige au-dessus des fleurs du printemps, tandis que beaucoup d'autres s'en vont voletant, les unes par-ci, les autres par-là ; ainsi des nefs et des baraques, des troupes sans nombre viennent se ranger, par groupes serrés, en avant du rivage bas, pour prendre part à l'assemblée. Parmi elles Rumeur, messagère de Zeus, est là qui flambe et les pousse à marcher, jusqu'au moment où tous se trouvent réunis ${ }^{17}$.

Le scholiaste insiste ici sur la vivacité de la comparaison des Grecs à des essaims d'abeilles, sur la disposition analogue en groupes compacts et l'ordre de l'armée comparable à celui de l'essaim : en appliquant aux deux domaines la notion de la phalange ( $\dot{\eta} \mu \grave{\varepsilon} v$ oũv $\varphi \alpha \lambda \alpha \gamma \gamma \eta \delta \dot{\nu} v$

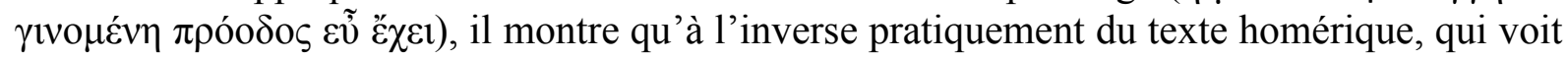
les hommes comme un essaim, lui-même se représente les abeilles comme groupées en phalanges, probablement à cause de l'emploi de عُ $\theta v \varepsilon \alpha$ pour $\sigma \mu \eta ́ v \varepsilon \alpha$ sur lequel porte sa première remarque. Il insiste ensuite sur un aspect de la comparaison qui ne nous semble pas vraiment pertinent, à savoir le fait que dans les deux domaines, les groupes sont armés : là encore il essaie de trouver des justifications pour la pertinence de la comparaison par toutes les caractéristiques de chaque terme, et comme plus haut pour l'avance en phalanges, il affirme que les abeilles sont «armées » (féminin $\hat{\omega} \pi \lambda \imath \sigma \mu \varepsilon ́ v \alpha l)$ comme les hommes, par leur dard ou aiguillon ( $\kappa \varepsilon ́ v \tau \rho o ı \varsigma)$. Pour nous évidemment, cette mention aurait presque tendance à amoindrir la valeur poétique de la comparaison. Si l'on regarde le texte homérique lui-même en se dégageant de la scholie, on voit que l'abondance des abeilles en groupes toujours

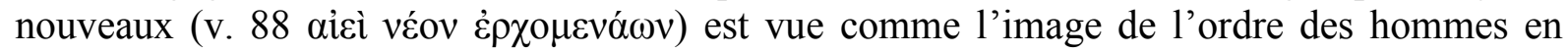

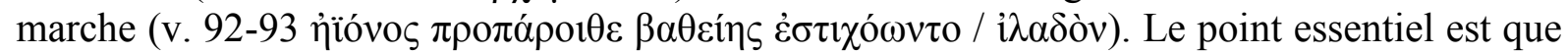
la comparaison ne vaut nullement pour un essaim de combattants, mais pour des hommes se rendant en ordre à l'assemblée : je n'oserais parler d'un essaim démocratique ou civil, mais en tout cas, ce qui intéresse le poète n'est sûrement pas ici l'ordre militaire. La précision du scholiaste semble donc à la limite du contresens. Pour la première comparaison de l'Iliade, l'essai n'est pas très concluant !

Le scholiaste semble avoir compris que les comparaisons homériques se situent entre deux mondes, celui du réel et celui des images, mais il a été victime ici de l'impression généralement répandue sur la prégnance du domaine de la guerre, qui lui a fait manquer la valeur de cette comparaison particulière, à partir de laquelle on pourrait peut-être parler d'une poétique du collectif dans l'Iliade, dont il existe d'autres exemples.

Dans plusieurs cas, les scholies renvoient d'un passage à un autre, montrant une méthode comparative et typologique dont nous retrouverons d'autres exemples.

Il. XVII comporte plusieurs exemples de comparaison des héros à un lion, avec une discussion dans les scholies portant sur le genre et les qualités respectives des mâles et femelles. Ainsi la scholie à XVII, 133-6 commente la comparaison d'Ajax combattant autour de la dépouille de Patrocle à un lion (mâle : $\lambda \dot{\varepsilon} \omega v$ ) défendant ses petits, en renvoyant à un autre passage du même chant, où il s'agit d'un animal femelle beaucoup plus faible, une

\footnotetext{
${ }^{17}$ Les traductions de l'Iliade sont celles de P. Mazon
} 
vache simplement désignée comme «mère » servant d'image à Ménélas auprès du même Patrocle mort :

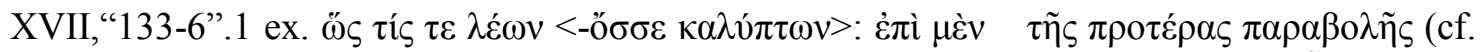

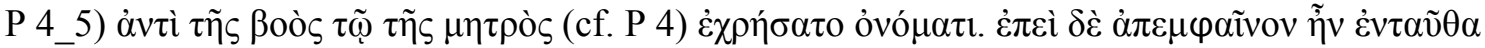

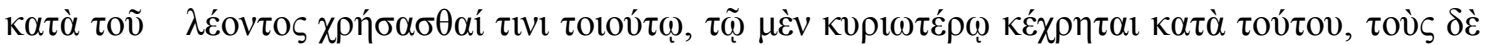

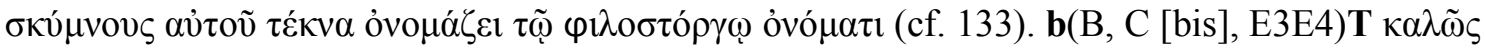

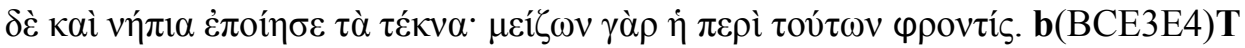

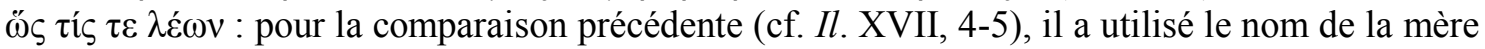
(cf. Il. XVII, 4) au lieu de celui de la vache, mais comme il aurait été absurde de s'en servir ici pour le lion, il s'est servi du nom le plus propre pour lui, et il a appelé ses petits ses enfants par ce nom plein d'affection; car le souci qu'il se fait pour eux est mieux dit par là.

Le texte homérique montre en effet Ajax comme un lion mâle protégeant ses petits :

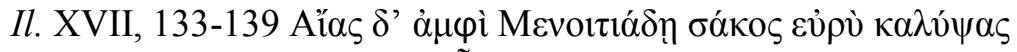

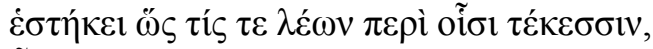

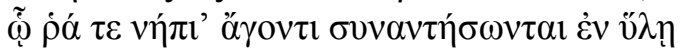

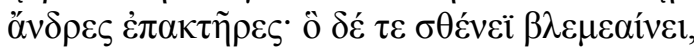

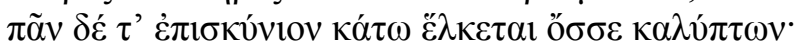

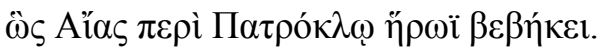

'A

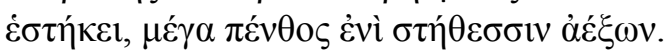

Ajax, lui, de son large écu couvre le fils de Ménœtios. Il se tient là, pareil à un lion protégeant ses enfants - il s'est rencontré avec des chasseurs, alors qu'il menait ses petits aux bois, et, enivré de sa force, il abaisse sur ses yeux - les couvrant entièrement - toute la peau de son front. Tel s'est dressé Ajax aux côtés du héros Patrocle. Près de lui se tient l'Atride, Ménélas chéri d'Arès, qui sent dans sa poitrine grandir un deuil immense.

Le début du chant montrait Ménélas protégeant le cadavre de Patrocle comme une vache avec

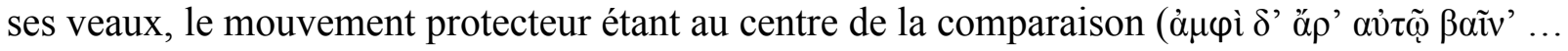

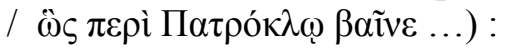

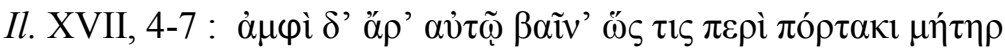

$\pi \rho \omega \tau$ о

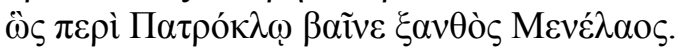

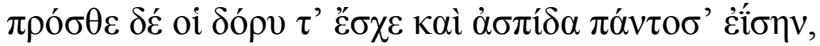

Il se poste à ses côtés, comme aux côtés d'une génisse fait sa mère gémissante - mère pour la première fois, hier encore ignorant l'enfantement -, ainsi aux côtés de Patrocle se poste le blond Ménélas. Il tient sa lance en avant, ainsi que son écu rond.

Les commentaires des scholiastes pour ce passage insistent sur le caractère affectif de la mère animale :

XVII."4a".1

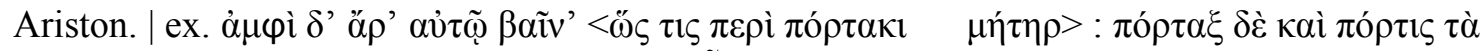

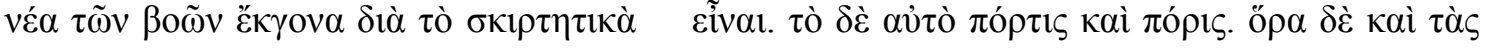

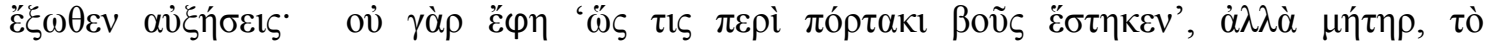

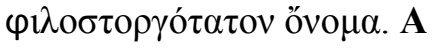

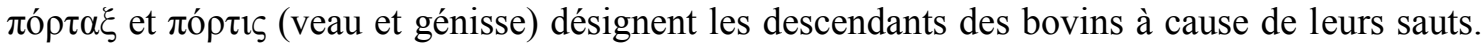

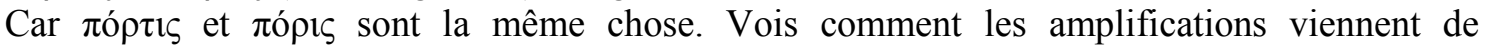
l'extérieur. Car il n'a pas dit «Comme une vache se tient près de son petit », mais «Comme une mère ... »: le mot est le plus chargé affectivement.

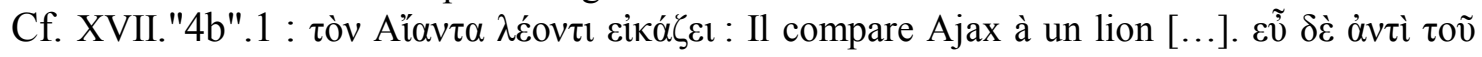




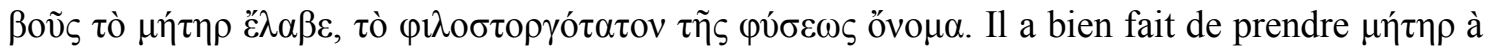
la place de $\beta$ oṽ $\varsigma$, c'est le nom le plus affectueux de la nature.

Les scholies au premier texte (Il., XVII, 4-7 dans l'ordre linéaire du texte) insistent sur le caractère maternel de la protection en mettant en valeur les qualités traditionnelles du féminin, mais surtout le fait que le poète n'emploie pas le nom habituel pour la vache, femelle du bœuf, mais le remplace par "mère " et ce, plus particulièrement dans le commentaire au

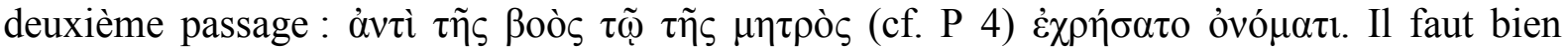

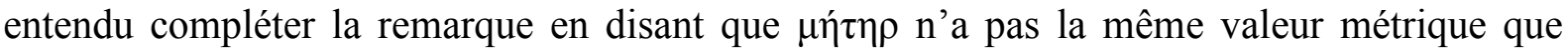
ßoṽ, mais les scholiastes, avec raison me semble-t-il, n'ont même pas invoqué cet argument. Les scholiastes semblent aussi assez généralement avoir été stimulés par l'emploi du composé $\pi \rho \omega \tau$ оóко $\zeta$, signifiant « qui enfante pour la première fois », effectivement redoublé dans le

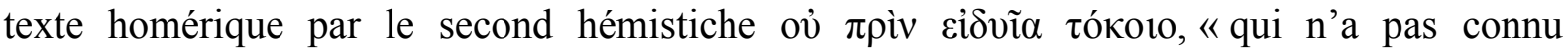
l'enfantement auparavant »; ils pensent que cet adjectif met en valeur la tendresse de l'animal, sa qualité affective ( $\varphi$ i $\lambda$ ó $\tau \tau$ o $\rho \gamma o v$ et même, dans un cas, le superlatif $\varphi 1 \lambda о \sigma \tau о \rho \gamma o ́ \tau \alpha \tau o v)$. En somme les scholiastes ont l'air de penser que la force d'Ajax est bien exprimée par la comparaison à un lion défendant ses petits, mais perd peut-être une partie de la valeur de tendresse maternelle exprimée par la comparaison précédente de Ménélas à une mère animale bien moins forte physiquement. Les comparaisons homériques impliquent pourtant parfois des lionnes; la femelle du lion aurait pu ici exprimer la force d'Ajax supérieure à celle de Ménélas, bien plus capable de protéger efficacement le cadavre de Patrocle, mais les scholiastes n’y ont apparemment pas songé.

Pourtant les comparaisons animales permettent parfois de poser le problème du " genre ». Ainsi en Il. XVIII, 318, la comparaison d'Achille gémissant à un(e) lion(ne) à la belle crinière

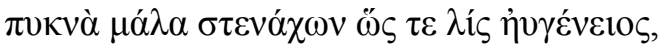

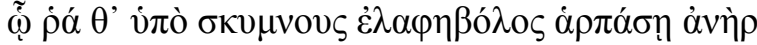

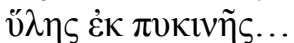

Il sanglote sans répit, tel un lion à crinière à qui un chasseur de biches a enlevé ses petits au fond d'une épaisse forêt...

suscite d'abondants commentaires des scholiastes, et l'un parle d'un lion mâle ( $\lambda \dot{\varepsilon}$ ov $\tau)$, mais deux commentateurs y voient une femelle :

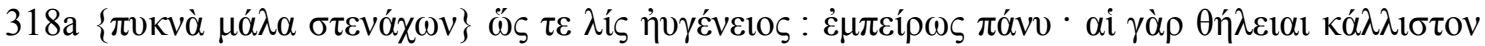

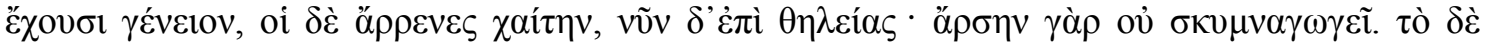

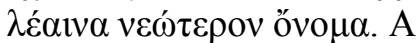

Tout à fait pertinent. En effet, les femelles ont une belle barbe, tandis que les mâles ont une crinière; il s'agit ici d'une femelle, car un mâle n'élève pas les petits. Le nom récent correspondant est leaina.

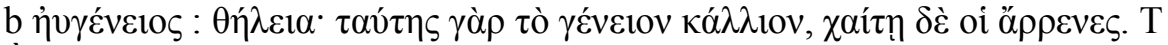

À la belle barbe : femelle; elle a une plus belle barbe, alors que les mâles se distinguent par la crinière.

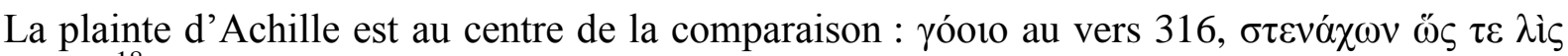

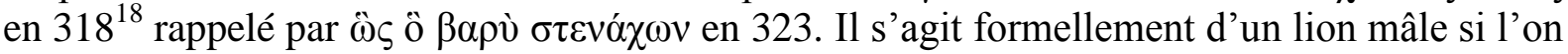

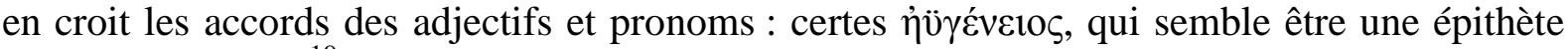

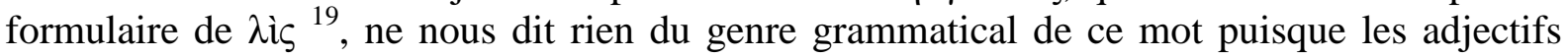

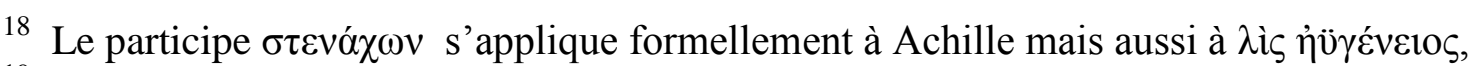

${ }^{19}$ Parmi les trois emplois homériques de $\lambda i \grave{s}$ au sens de lion (deux exemples dans l'Odyssée

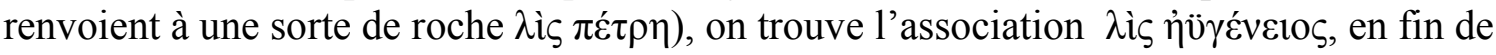




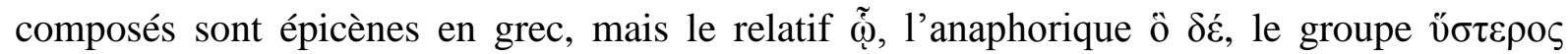
$\dot{\varepsilon} \lambda \theta \omega ́ v$ et le participe $\dot{\varepsilon} \rho \varepsilon v v \tilde{\omega} v$ sont tous des masculins sans ambiguïté. Cela suffit-il à exclure

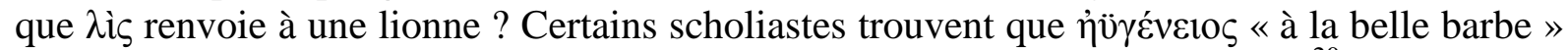
convient à la femelle alors que les lions mâles se caractérisent par une crinière ${ }^{20}$. Cela semble même évident pour Mark Edwards, commentateur de ce passage ${ }^{21}$. Mais la présence de la crinière n'exclut pas celle d'une barbe et le poète a pu s'attacher à un détail physique de

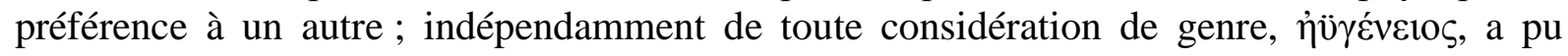
s'associer spécifiquement à $\lambda \grave{i} \varsigma$, mot rare, alors qu'un jeu d'épithètes variées était disponible pour $\lambda \dot{\varepsilon} \omega v$. En fait, il faut conclure que le genre grammatical est masculin pour $\lambda \dot{\varepsilon} \omega v$ comme pour $\lambda i \varsigma$, mais que le poète peut entendre une femelle protégeant ses petits dans les deux cas, comme le dit Fränkel dans le passage auquel renvoie la première note d'Edwards (citée ici n. 19).

La comparaison entre deux passages comportant des images voisines entraîne parfois dans les scholies le rejet d'un passage. Le scholiaste montre alors que sa connaissance du texte homérique l'amène à penser qu'il y a un seul cas où la comparaison est justifiée, à sa place, alors que l'autre passage manifesterait une interpolation : ce sont de tels passages qui ont donné naissance aux idées "séparatistes », et les ont peut-être justifiées au moins en partie. Pour $I l$. VI, 506-511 (la comparaison concerne Pâris, qui s'est apprêté pour le combat, v. 503-505):

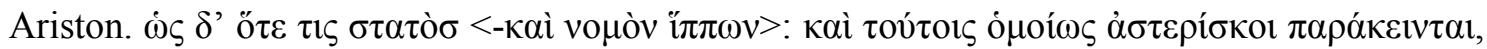

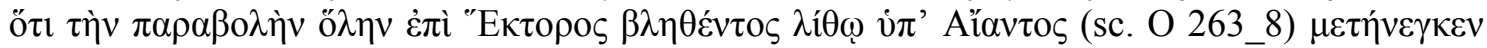
$\dot{\varepsilon} \nu \tau \varepsilon \tilde{\theta} \theta \varepsilon v$.

Comme lorsqu'un cheval à l'étable... : les astérisques se trouvent pareillement en face de ces vers parce que le poète a emprunté l'ensemble de la comparaison à celle d'Hector blessé d'une pierre par Ajax (sc. XV, 263-268).

Ce passage est ainsi rejeté au profit de $I l$. XV, 263-269 (la comparaison s'applique alors à Hector), le scholiaste pense que la comparaison de Pâris à un "cheval à l'étable » a été « empruntée » ( $\mu \varepsilon \tau \eta ́ v \varepsilon \gamma \kappa \varepsilon v)$ à celle d'Hector :

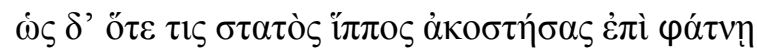

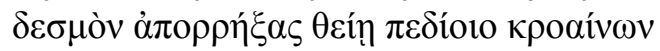

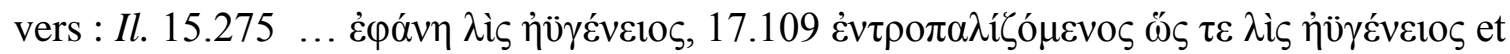

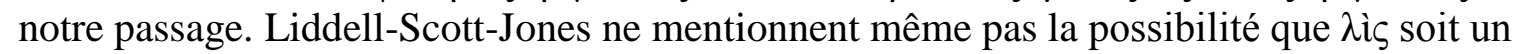
féminin.

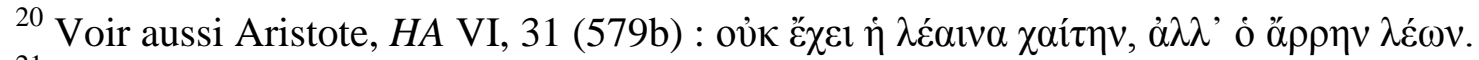

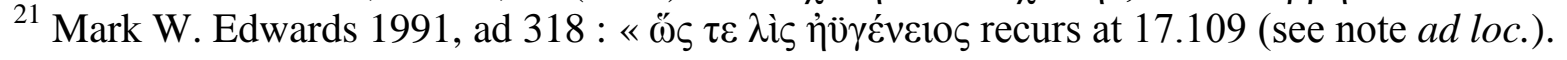

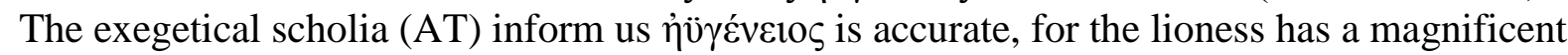

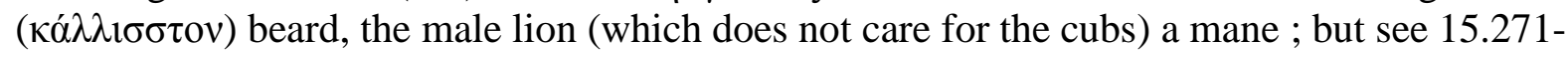
6n. On $\lambda$ is see 17.133-6n. » Le même Mark Edwards note pourtant dans la note au chant 17 à laquelle renvoie sa dernière phrase citée qu'il s'agit alors d'un lion mâle avec la même thématique de la défense des petits : «Zenodotus omitted these lines (which were not in the Chian text ; see Apthorp,, Manuscript Evidence 102-4, n. 11) on the grounds that male lions do not lead their cubs about ; familiarity with the habits of lions had led him to alter the text elsewhere (see 13.198-200n., and cf. Pasquali, Storia 229). Aristarchus (Did/A) correctly

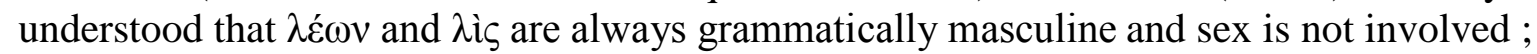
see Fränkel, Gleichnisse 92-3, on the gender of female animals. » 


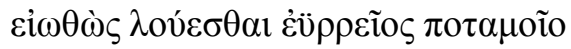

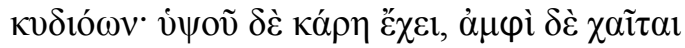

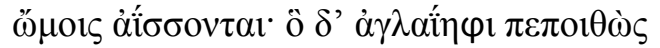

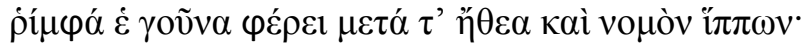

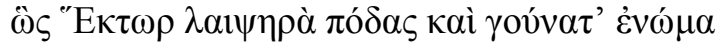

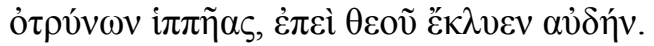

Tel un étalon, trop longtemps retenu en face de la crèche où on l'a gavé d'orge, soudain rompt son attache et bruyamment galope dans la plaine, accoutumé qu'il est à se baigner aux belles eaux d'un fleuve. Il se pavane, il porte haut la tête ; sur ses épaules voltige sa crinière, et, sûr de sa force éclatante, ses jarrets promptement l'emportent vers les lieux familiers où paissent les cavales. Tel Hector, rapide, joue des pieds, des jarrets, pour aller stimuler ses meneurs de chars dès l'instant où il a ouï la voix du dieu.

Il est intéressant de constater que l'ordre linéaire du texte de l'Iliade ne semble avoir pour le scholiaste aucune pertinence ici ; la comparaison adaptée à Hector au chant XV a pu selon lui entraîner secondairement son application à Pâris au chant VI. En revanche, la scholie à la comparaison du chant II citée plus haut semble implicitement attacher de l'importance à cet ordre, sans parler des deux scholies au XVII qui comparent deux comparaisons dans le même chant. Mais il ne s'agit pas alors de l'ensemble de l'épopée. Le problème des scholiastes est celui de deux images analogues pour des personnages et des contextes différents : pour eux, cela suppose qu'un passage est authentique, l'autre imité. Il s'agit donc de déterminer le passage unique dans lequel la comparaison est pertinente et d'exclure la possibilité de multiples usages du même modèle de comparaison.

Une scholie au chant XV défend pourtant la position inverse, montrant que pour chaque passage, tel ou tel commentateur est prêt à développer une argumentation ad hoc en fonction de son interprétation du passage et du personnage en question :

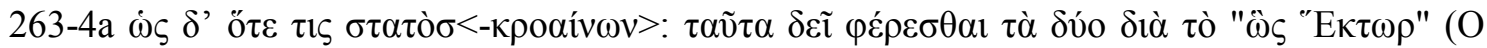

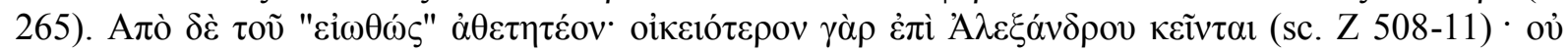

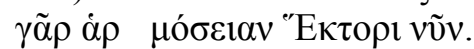

Comme lorsqu'un cheval ... : il faut enlever ces deux vers à cause de «ainsi Hector »; il faut

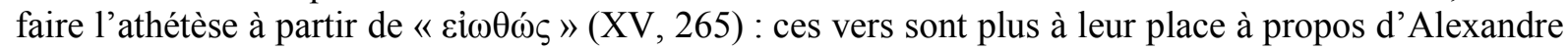
(sc. VI, 508-511); ils ne sauraient convenir maintenant à Hector.

Il me semble que l'on peut proposer plutôt une hypothèse dans le cadre de l'Oral Poetry : des images différentes du ou de héros différents peuvent se rencontrer dans différents contextes où elles donnent au récit une dimension de profondeur, une couleur poétique, sans nécessiter un choix du meilleur contexte aux dépens des autres occurrences ${ }^{22}$. Mais cela n'est nullement le point de vue des scholies.

Du point de vue - moderne - de l'anthropologie des images évoqué incidemment plus haut, on pourrait surtout suggérer que la comparaison d'Hector à un cheval à l'arrêt au chant XV prépare les comparaisons du chant XXII (en particulier 162-166 : la poursuite d'Hector par Achille entraîne une comparaison à une course de chevaux autour de la ville, qui n'a pas déclenché la verve des scholiastes, semble-t-i ${ }^{23}$ ). Dans le contexte du chant XV lui-même,

\footnotetext{
${ }^{22}$ Cet exemple précis est d'ailleurs cité par W. C. Scott - dont le point de vue central est de montrer que la technique homérique des comparaisons correspond à une composition orale - : il renvoie au commentaire de Leaf et au principe des analystes sans mentionner les scholies (The Oral Nature of the Homeric Simile, p. 6).

${ }^{23}$ Mais Eustathe l'a remarqué, il insiste sur l'inégalité des deux «chevaux » de l'image, Achille vainqueur

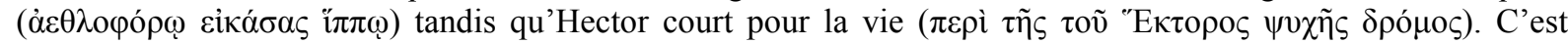
d'autant plus remarquable que l'évêque byzantin reproduit d'ordinaire l'essentiel des scholies antérieures : ici, il ajoute sa remarque personnelle.
} 
elle suit d'ailleurs un discours d'Apollon à Hector évoquant les meneurs de chars des Troyens et leurs chevaux rapides (vers 257 et 258).

Cet exemple suscite l'envie de voir ce que les commentateurs disent dans d'autres cas de comparaisons formulaires. La multiplicité des comparaisons entre un héros et un lion dont il a été question plus haut montre bien que le problème aurait pu être posé dans les scholies.

Il semble relativement rare que les scholies constatent la coalescence de plusieurs figures dans un même passage, ainsi pour Il. II, 382, où la présence d'un homeotéleute combiné à une anaphore est soulignée par un scholiaste, avec $\sigma v v \varepsilon ́ \pi \lambda \varepsilon \xi \varepsilon \sigma \chi \eta \dot{\mu} \mu \tau \alpha$ (« tresser les figures ») qui me paraît remarquable, même s'il nous fait sortir du cadre des comparaisons et métaphores que nous avons choisi :

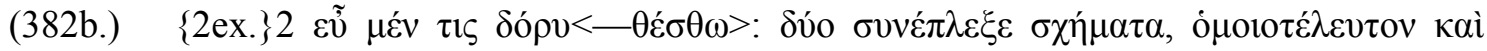

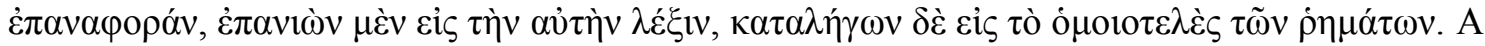
$\mathrm{b}(\mathrm{BCE} 3) \mathrm{T}$

Il a tressé deux figures, l'homéotéleute et l'épanaphore, en revenant sur la même expression et en terminant par une fin de mot identique.

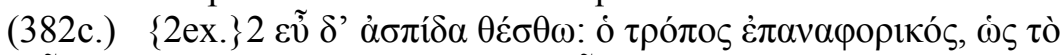

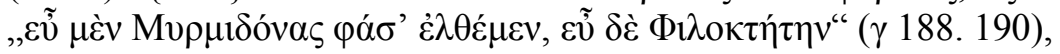

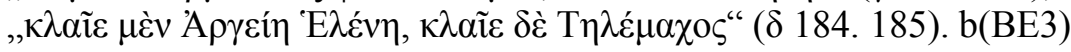

Le trope est une épanaphore comme dans les vers suivants : Od. III, 188/190 et IV, 184/185.

Il me semble intéressant aussi de remarquer que la Rhétorique d'Aristote et le traité Du style de Démétrius admirent de même Homère pour de telles superpositions de figures dans le passage du Catalogue où il est question du beau Nirée, dont le nom revient à trois reprises dans le texte homérique. Les deux passages, visiblement redondants l'un par rapport à l'autre, laissent penser que Démétrius s'est largement inspiré de son prédécesseur :

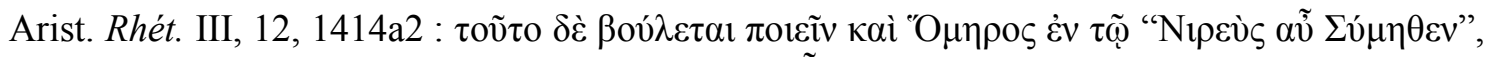

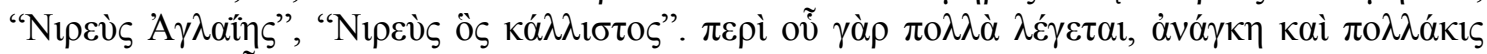

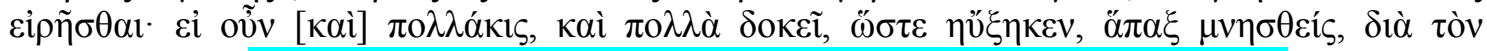

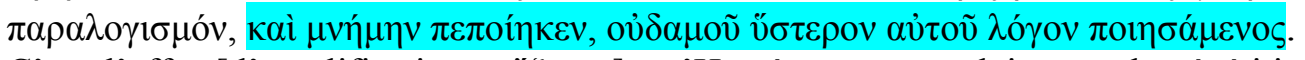

C'est l'effet [d'amplification, $\alpha$ ' $\xi \eta \sigma ı]$ qu'Homère veut produire par la répétition : « Nirée, venu de Symé ... », " Nirée, fils d'Aglaé ... », « Nirée, qui était le plus beau ». En effet, celui dont on dit plusieurs choses est nécessairement nommé plusieurs fois; si donc on le nomme plusieurs fois, il semble qu'il accomplisse plusieurs exploits; le poète a donc, grâce à ce paralogisme, accru l'importance du personnage, tout en ne le mentionnant qu'en un seul passage, et il en a assuré la mémoire, sans jamais faire mention de lui par la suite (traduction personnelle) ${ }^{24}$.

\footnotetext{
${ }^{24}$ Nirée est un personnage très secondaire de l'Iliade, dont les exploits n'ont guère d'importance dans le récit, Aristote le dit très justement, sans expliquer à quoi pouvait correspondre cette insistance. Comparer avec le Ps.Démétrios, Du style, §61-62 : «Quant à Nirée, mince personnage s'il en fut, à la fortune encore plus mince (trois navires et une poignée d'hommes), Homère l'a fait grand, grande sa fortune et nombreuse sa maigre suite, par l'usage d'une double figure, faite du mélange de l'épanaphore et de la disjonction : car Nirée, dit-il, amenait trois navires, Nirée le fils d'Aglaea, Nirée le plus bel homme venu sous Ilion... La remontée de l'expression (épanaphore) au même mot, Nirée, et la disjonction, font l'effet d'une abondance de biens au lieu des deux ou trois réels. (62) Aussi, bien que Nirée ne soit nommé qu'une fois à peine au cours de l'action, nous nous souvenons de lui autant que d'Achille ou d'Ulysse dont il est pourtant question presque à chaque vers. La raison en est le pouvoir de la figure. Si Homère avait dit: "Nirée, fils d'Aglaea, amenait de Symé trois navires", cela serait presque revenu à passer Nirée sous silence. Il en va, en littérature, comme dans les banquets, où une petite quantité de mets disposée d'une certaine façon fait croire à une grande quantité. » (tr. P. Chiron)
} 
Le problème le plus intéressant à mes yeux, celui de la frontière entre métaphore et comparaison, est rarement abordé par les scholiastes autrement que de manière très allusive, mais permet de réfléchir sur leurs pratiques ${ }^{25}$.

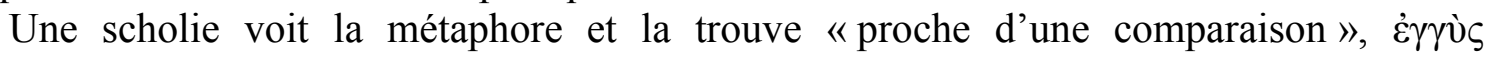
$\pi \alpha \rho \alpha \beta \circ \lambda \tilde{\eta} \varsigma \dot{\eta} \mu \varepsilon \tau \alpha \varphi \circ \rho \alpha ́$, une autre remarque sa particularité dans le cas d'Il. XIII, 339a :

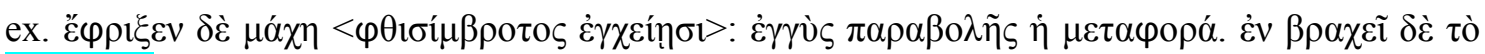

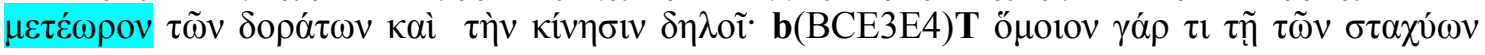

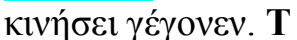

\section{Le combat se hérissa : la métaphore est proche d'une comparaison ; avec concision elle montr \\ mouvement. Il est en effet semblable au mouvement des épis. \\ XIII."339b".1

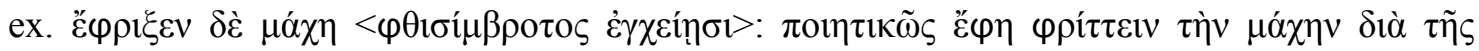

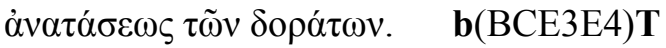

Le combat se hérissa: (le poète) dit poétiquement que le combat se hérisse à cause du redressement des lances.

\section{XIII."339c".1 dit à peu près la même chose en plus condensé :}

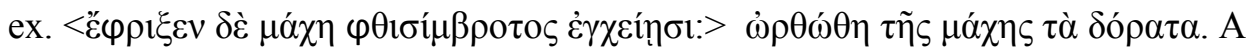
Le combat se hérissa : les lances du combat se dressèrent.

La métaphore du frisson qui passe sur les armées en bataille évoque pour le premier commentaire le passage du vent sur les épis de blé, comparaison qui a effectivement des parallèles chez Homère. En l'occurrence, si l'on regarde le contexte immédiatement antécédent du vers 339, on remarque que les vers 334 à $338^{26}$ sont justement une grande comparaison du combat à une tempête qui disperse de la poussière : ce n'est nullement une métaphore exceptionnelle, mais une métaphore qui prolonge une comparaison sous forme synthétique. Les spécialistes modernes ne semblent pas plus que les anciens avoir vu le phénomène: les uns commentent la comparaison ${ }^{27}$, les autres la métaphore comme le scholiaste $^{28}$, mais ne voient pas le contexte d'ensemble - à moins que ce ne soit ce que voulait

\footnotetext{
${ }^{25}$ On ne peut que regretter qu'Aristote, qui parle de la comparaison et de la métaphore dans la Rhétorique quelques paragraphes avant celui sur Nirée, et dit bien que la comparaison est réussie quand elle touche à la métaphore (1413) en donnant des exemples peu convaincants, n'ait pas analysé de près les comparaisons homériques. Sur cette relative déception, voir W. B. Stanford, Greek Metaphor, p. 28-29, qui souligne toutefois deux passages dans lesquels le Stagirite exprime son admiration pour certaines métaphores, Poét. 22, 59a et Rhét. III, 10, 1410-1411, où Homère n'est pas concerné. Son exemple le plus probant à mes yeux est le suivant : «Des quatre sortes de métaphores, les plus réputées sont celles qui se fondent sur une analogie, comme Périclès disait que la jeunesse morte pendant la guerre avait disparu de la cité comme si l'on avait retranché le printemps de l'année. »

${ }^{26}$ Ces vers sont ainsi traduits par F. Mugler: «Tels, sous le fouet de la tempête, on voit les vents sonores, / toutes les fois que la poussière abonde sur les routes, / la ramasser pour en former un nuage poudreux : / tel le combat ne fit plus qu'un seul bloc, et tous brûlèrent / de s'égorger dans la mêlée avec le bronze aigu. »

${ }^{27}$ E. Fraenkel, Die Homerischen Gleichnisse, p. 23 ; W. C. Scott, The oral Nature of the Homeric Simile, p. 65.

${ }^{28}$ W. B. Stanford, Greek Metaphor, p. 28, dont la remarque vient de Démétrius, Du style, §82 : «Certaines choses sont dites plus clairement et plus proprement dans les métaphores que dans les mots propres eux-mêmes, comme pour "la bataille se hérissa". En effet, on ne le dirait pas avec plus de vérité et de clarté en passant par les mots propres. Le poète a désigné l'agitation des lances et le son qui se produit lentement par un combat qui est continuellement en train de se hérisser, et en même temps il s'est emparé de la métaphore en puissance dont il a été question plus haut, en disant que le combat se hérisse comme un animal (c'était ma traduction, voici celle de P. Chiron vous pouvez faire le remplacement si vous préférez: «Il y a meme certains choses qui s'expriment plus clairement et plus proprement par métaphore qu'avec les termes propres eux-mêmes. Par exemple: et la bataille frissonna. Si l'onchangeait la phrase à l'aide des termes propres, on n'obtiendrait pas une expression plus vraie ni plus Claire. C'est le frémissement des lances et le bruit sourd et continu qui en résulte que le Poète
} 


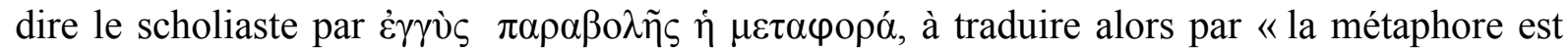
proche de la comparaison » (en entendant celle qui précède immédiatement), mais l'absence d'article avec $\pi \alpha \rho \alpha \beta o \lambda \tilde{\eta} \varsigma$ ne plaide pas dans ce sens.

Un peu plus loin dans le même chant XIII, un scholiaste commente en parlant d'allégorie et d'une comparaison «mixte » et dit que le poète se sert des éléments du combat comme métaphore : le scholiaste embarrassé évoque une comparaison tout en notant qu'elle ne suit pas le schéma corrélatif habituel mais fonctionne en réalité comme une métaphore? 29 :

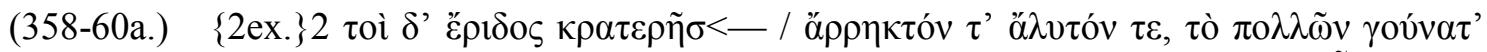

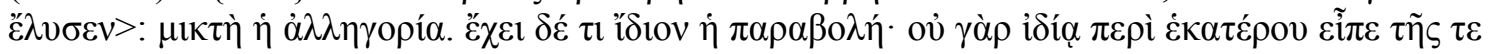

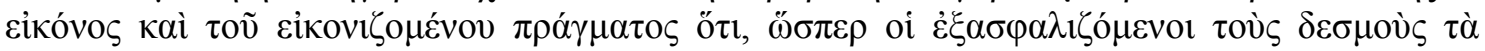

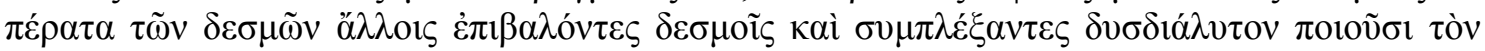

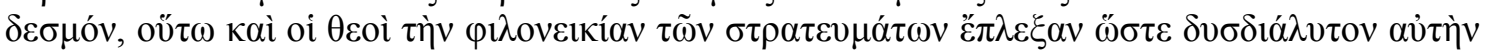

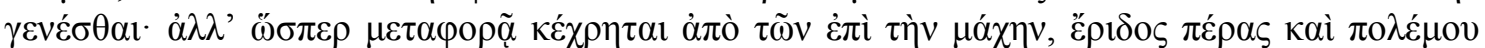

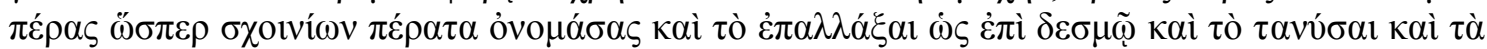
$\dot{\varepsilon} \xi \tilde{\eta} \varsigma$ ỏvó $\mu \alpha \tau \alpha$. traduire ou bien supprimer le texte de la scholie et se contenter de la résumer

Dans un autre cas, en commentant l'interversion de l'ordre habituel des termes dans une comparaison, un scholiaste semble faire par défaut une typologie des comparaisons homériques habituelles. Le texte commenté est celui de Iliade, II, 207-210 :

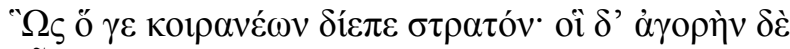

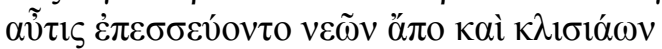

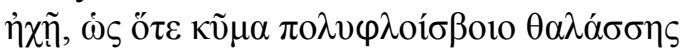

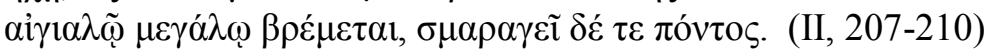

Ainsi il parle en chef et remet l'ordre au camp; et, de nouveau, des nefs et des baraques, l'armée accourt à l'assemblée. Le fracas en est tout pareil à celui des flots d'une mer bruyante, qui mugit au long d'un rivage immense, cependant que gronde le large.

Trois Les scholiastes proposent des les commentaires voisins suivant pour ces vers :

II."207-10".1

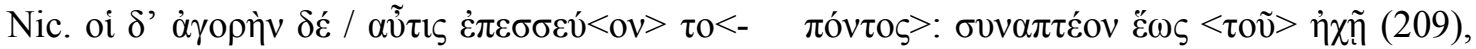

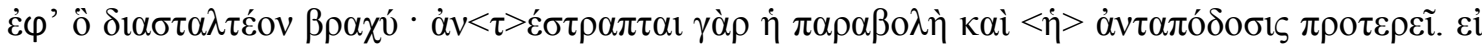

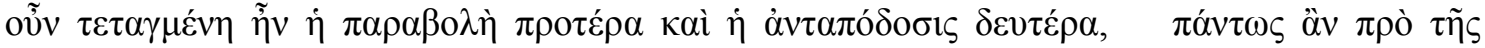

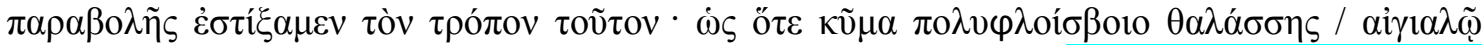

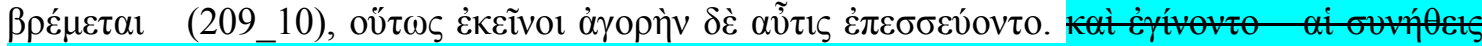

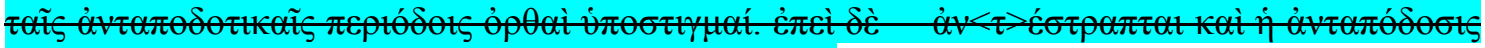

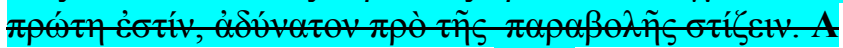

Il faut lier jusqu'au mot ỉxñ,, après lequel il faut marquer une brève séparation. En effet la comparaison est dans l'ordre inverse et l'antapodose (sc. le développement du comparé) vient en premier. Si donc la comparaison était placée en premier et l'antapodose en second, nous aurions ponctué de la manière suivante tout à fait devant/avant la comparaison

II."209a1".1

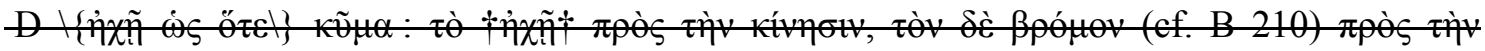
èveńxnow. T

HI."209a2".1

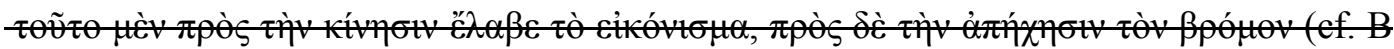
210). b(BCE3)

a appelés bataille frissonnante; en meme temps, il s'est en quelque sorte empire de la métaphore par animation don't nous parlions plus haut en disant que la bataille frissonnait, comme un être vivant. »

${ }^{29}$ Scholie à Il. XIII, 358-360a. 
Effectivement, le récit initial entraîne une comparaison à la mer en furie qui pourrait tenir en

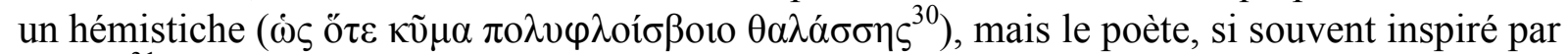
la $\mathrm{mer}^{31}$, semble s'être laissé aller à développer davantage la comparaison et à permuter l'ordre habituel du comparant et du comparé.

J'ai déjà abordé le passage d'Iliade III, 60-65 dans le cadre du colloque $\Sigma \chi \tilde{\eta} \mu \alpha / F i g u r a:$ que l'on me permette d'y revenir ici à cause de son importance.

Commençons par le texte originel, Il. III, 60-65 :

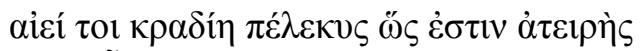

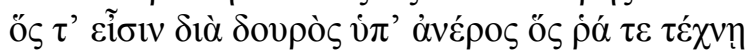

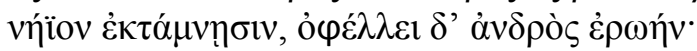

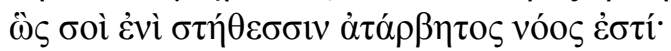

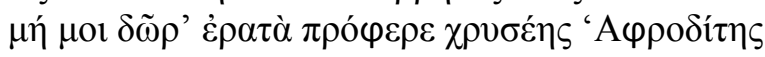

Ton cœur à toi toujours est inflexible : on croirait voir la hache qui entre dans le bois, quand, aux mains de l'artisan taillant la quille d'une nef, elle aide à l'effort de l'homme. Ton cœur est aussi ferme au fond de ta poitrine. Ne me reproche pas pourtant les dons charmants de l'Aphrodite d'or.

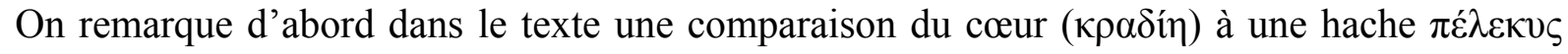

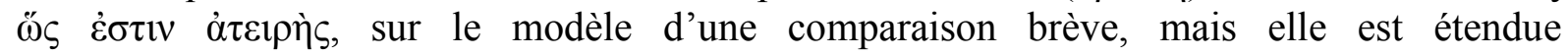
secondairement par une relative elle-même développée sur deux vers (61-62). Le vers 63 montre que le poète s'est ensuite senti dans le cadre typologique de la grande comparaison, commençant le vers par le ôऽ que l'on appelle «résomptif», mais avec l'application de

Le passage a suscité plusieurs scholies. Dans la scholie au vers 60, le scholiaste se tire d'une

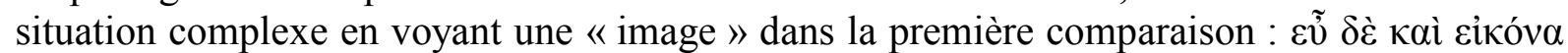

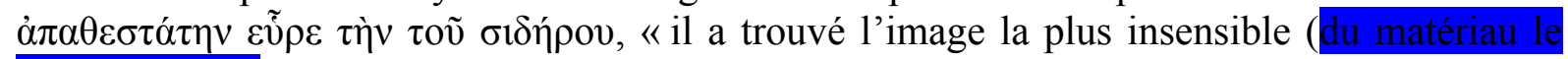
plus résistant ?), celle du fer », ce qui nous a amenés à faire entrer l'ensemble dans l'étude des attestations $\mathrm{du}$ « cœur de fer » et de ses variantes (de bronze ou de pierre).

Dans la scholie au vers 63 , le même - ou un autre scholiaste plutôt - commente le deuxième terme en y voyant la superposition de deux images :

III." $63 b " .1$

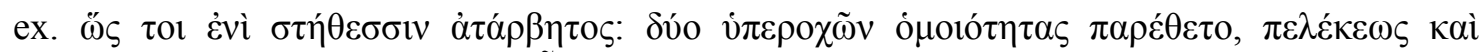

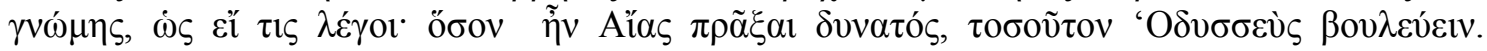
b(BCE3) $\mathbf{T}$

il a juxtaposé deux comparaisons d'excellence, celle de la hache et celle de la pensée, comme si l'on disait : "autant Ajax était bon pour agir, autant Ulysse pour décider" ${ }^{32}$.

On voit dans quelle perplexité nous nous trouvons actuellement. Faute de conclure, on pourrait dire que le problème de la relation entre le texte homérique et ses commentateurs apparaît dans sa complexité... Il faudra essayer de regarder systématiquement les commentaires disponibles pour chaque comparaison. Mais il est fort difficile de tenir compte de tous, ne serait-ce qu'à cause de la difficulté de visualiser les différents commentaires pour

\footnotetext{
${ }^{30}$ Cf. le même second hémistiche en Il. I, 34 ; II, 209 ; VI, 247 ; IX, 182 ; XIII, 798 ; XXIII, 59 ; Od. XIII, 85 et 220.

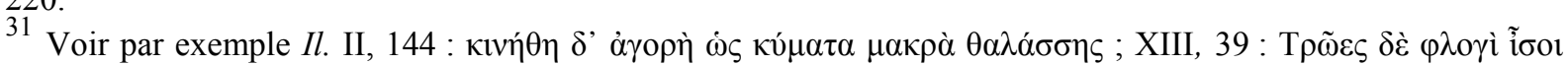

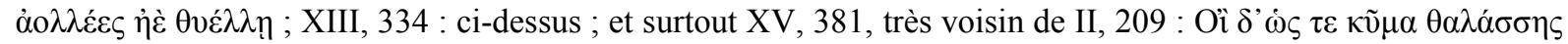

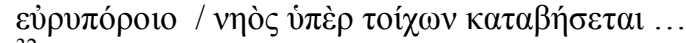

${ }^{32}$ On voit que la suite du raisonnement n'est pas évidente.
} 
chaque texte. L'exemple des comparaisons répétées et des commentaires contradictoires entre eux nous semble une voie intéressante pour préparer la suite.

Les analyses des scholies sont certes moins profondes que celles du PseudoPlutarque $^{33}$, mais elles vont dans le même sens du rapport entre comparé et comparant par rapport au contexte, et elles invitent à poursuivre la recherche dans le cadre d'un projet collectif.

\footnotetext{
${ }^{33}$ Voir dans le présent volume l'article correspondant à la belle communication d'Hélène Fuzier.
} 


\section{Bibliographie}

Sources $^{34}$

Dindorf, Wilhelm (éd.), Scholia graeca in Homeri Odysseam, Amsterdam, 1962, réimpression de l'édition d'Oxford, 1855.

Erbse, Hartmut (éd.), Scholia graeca in Homeri Iliadem (scholia vetera), 5 vol., Berlin, 19771988.

Études sur les scholies

Clay, Diskin, «The Theory of the literary persona in Antiquity », MD, 40, 1998, p. 9-40.

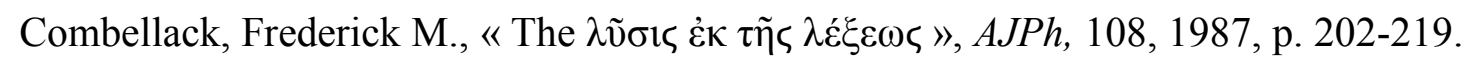

De Jong, Irene J.F., « Gynaikeion ethos: misogyny in the Homeric scholia », Eranos, 89, 1991, p. 13-24.

Dickey, Eleanor, Ancient Greek Scholarship. A Guide to Finding, Reading, and Understanding Scholia, Commentaries, Lexica and Grammatical Treatises, from their Beginnings to the Byzantine Period, Oxford, Oxford University Press, 2007.

Heath, Michael, «The Homeric Scholia », in Unity in Greek Poetics, chap. 8, Oxford, 1989, p. 102-123.

Kennedy, George A., « Ancient Antecedents of Modern Literary Theory », AJPh, 110, 1989, p. $492-498$.

Lamberton, Robert, « Homer in Antiquity », in Morris Ian et Powell Barry (éds.), A New Companion to Homer, Leiden, Brill, 1997, p. 33-54.

Lamberton, Robert et Keaney, John J. (éds.), Homer's Ancient Readers : the Hermeneutics of Greek Epic's Earliest Exegetes, Princeton, 1992.

Montanari, Franco, Studi di filologia omerica antica I et II, Pise, Giardini, 1979 et 1995.

Montanari, Franco (éd.), Omero : gli aedi, i poemi, gli interpreti, Florence, Scandicci, 1998.

Montanari, Franco (éd.), Omero Tremila anni dopo. Atti del Congresso di Genova, 6-8 luglio 2000, Rome, Ed. di Storia e Letteratura, 2002, $2009^{2}$.

Nagy, Gregory, « Homeric Scholia », in Morris Ian et Powell Barry (éd.), A New Companion to Homer, Leiden, Brill, 1997, p. 101-122.

Nannini, Simonetta, Omero e il suo pubblico. Nel pensiero dei commentatori antichi, Rome, Edizioni Dell'Ateneo, 1986.

Pfeiffer, Rudolf, History of Classical Scholarship, Oxford, 1968.

Rengakos, Antonios, « The Hellenistic Poets as Homer's Critics », in Montanari Franco (éd.), Omero Tremila anni dopo, p. 143-157.

Richardson, Nicholas J., « Literary Criticism in the Exegetical Scholia to the Iliad : a Sketch », CQ, 30, 1980, p. 265-287.

Richardson, Nicholas J., « La lecture d'Homère par les Anciens », Lalies, 10, 1988/89, p. 293-327.

\footnotetext{
${ }^{34}$ Éditions des scholies servant de base à la version numérisée dans le $T L G$.
} 
Schmidt, Martin, «The Homer of the Scholia : What is explained to the reader ? », in Montanari Franco (éd.), Omero Tremila anni dopo, p. 159-183.

Van Der Valk, Marchinus, Researches on the Text and Scholia of the Iliad, Leiden, 1963.

Wilson, Nigel G., « Scoliasti e commentatori », SCO, 33, 1983, p. 83-112.

Autres études

Bonnafé, Annie, "Quelques remarques à propos des comparaisons homériques de l'Iliade. Critères de classification et études statistiques », $R P h, 57,1983$, p. 79-97.

Casevitz, Michel, «Étude lexicologique. Du schèma au schématisme », in Maria Silvana, Chiron Pierre et Noël Marie-Pierre (éd.), Skhèma/Figura, p. 15-29.

Celentano, Maria Silvana, Chiron, Pierre et Noël Marie-Pierre (éd.), Skhèma/Figura. Formes et figures chez les Anciens, rhétorique, philosophie, littérature, Paris, Éditions Rue d'Ulm, «Études de littérature ancienne » 13, 2004.

Crubellier, Michel et Pellegrin, Pierre, Aristote, le philosophe et les savoirs, Paris, Seuil, 2002.

Durante, Marcello, « ЕПЕА ПТЕРОЕNTA. La parola come "cammino" in immagini greche e vediche », Rend. Acad. dei Lincei, Classe di Scienze Morali, storiche e filologiche, 13, 1958, p. 3-14 [trad. all. : "Epea pteroenta. Die Rede als 'Weg' in griechischen und vedischen Bildern », in Schmitt Rüdiger (éd.), Indogermanische Dichtersprache, Darmstadt,

Wissenschaftliche Buchgesellschaft, 1968, p. 242-260].

Edwards, Mark W., The Iliad : A Commentary, General editor G.S. Kirk, volume V : books 17-20, Cambridge, 1991.

Fränkel, Hermann, Die Homerischen Gleichnisse, Göttingen, 1921, 2. unveränderte Auflage, mit einem Nachwort und einem Literaturverzeichnis von Ernst Heitsch, Göttingen, 1977.

Fuchs, Catherine, La Paraphrase, Paris, 1982.

Fuchs, Catherine, Paraphrase et énonciation, Paris-Gap, 1994.

Lee, Dionys J.N., The Similes of the Iliad and the Odyssey compared, Melbourne, Melbourne University Press, 1964.

Létoublon, Françoise, « Epea pteroenta », Oral Tradition, 14, 1999, p. 321-335.

Létoublon, Françoise et Montanari, Franco, «Les métaphores homériques : l'exemple du "cœur de fer" », in Skhèma/Figura, p. 31-46.

Létoublon, Françoise, « Comparaisons et métaphores homériques : un formulaire traditionnel ou un art poétique? », Aevum Antiquum, n.s. 2005, p. 117-134 (publié en 2009).

Lonsdale, Steven H., Creatures of Speech : lion, herding and hunting similes in the Iliad, Stuttgart, 1990.

Nagy, Gregory, Pindar's Homer. The Lyric Possession of an Epic Past, Baltimore, The Johns Hopkins University Press, 1990.

Parry, Adam (éd.), The Making of Homeric Verse. The Collected Papers of Milman Parry, Oxford, Clarendon Press, 1971.

Parry, Milman, L'épithète traditionnelle dans Homère. Essai sur un problème de style homérique, Paris, Les Belles Lettres, 1928. 
Parry, Milman, Les Formules et la métrique d'Homère, Thèse complémentaire, Paris, 1928.

Parry, Milman, «The Homeric Metaphor as a Traditional Poetic Device », TAPhA, 62, 1931, p. XXIV [repris in Parry, Adam (éd.), The Making of Homeric Verse, Oxford, Clarendon Press, 1971, p. 419] (résumé d'un article en projet).

Parry, Milman, « The Traditional Metaphor in Homer », CP, 28, 1933, p. 30-43 [repris in Parry, Adam (éd.), The Making of Homeric Verse, Oxford, Clarendon Press, 1971, p. 365 375].

Rengakos, Antonios, « Apollonius Rhodius as a Homeric scholar », in Papanghelis Theodore D. et Rengakos Antonios (éd.), A Companion to Apollonius Rhodius, Leiden, Brill, 2001, p. 193-216.

Russell, Donald A. et Winterbottom, Michael (éds.), Ancient Literary Criticism. The principal texts in new translations, Oxford, 1972.

Sandoz, Claude, Les Noms grecs de la forme. Étude linguistique, Berne, 1982 (thèse de l'universté de Neuchâtel, 1971).

Schmitt, Rüdiger (éd.), Indogermanische Dichtersprache, Darmstadt, Beck, «Wege der Forschung »165, 1968.

Schnapp-Gourbeillon, Annie, Lions, héros, masques : les représentations de l'animal chez Homère, Paris, Maspero, 1981.

Scott, William C., The Oral Nature of the Homeric Simile, Leiden, Brill, « Mnemosyne Supplement » 28, 1974 [digital reissue, Dartmouth College Library, Hanovre, 2009].

Stanford, William B., Greek Metaphor. Studies in Theory and Practice, Oxford, B.

Blackwell, 1936. 